\title{
Updates in ASIA Examination: Definitions
}

\author{
Belgin ERHAN ${ }^{1,2}$ \\ 'Istanbul Physical Medicine Rehabilitation Training and Research Hospital, Istanbul, Turkey \\ ${ }^{2}$ Istanbul Aydın University, Health Services Vocational School of Higher Education, Istanbul, Turkey
}

\begin{abstract}
The most sensitive way of evaluating a spinal cord injury $(\mathrm{SCl})$ patient is to perform a standardized neurological examination. The standards that were developed for this purpose, provide an accurate communication between professionals dealing with $\mathrm{SCl}$ patients and also standardize scientific research protocols. In this article definitions and terms that are included in the International Standards for Neurological Classification of Spinal Cord Injury (ISNCSCI) booklet, which was revised in 2011 by American Spinal Injury Association (ASIA) and accepted by International Spinal Cord Society (ISCOS), will be discussed.

Keywords: ASIA definitions, neurological level of injury, incomplete injury, complete injury, zone of partial preservation
\end{abstract}

\section{Introduction}

The most sensitive way of evaluating a patient who sustained a spinal cord injury is to perform a standardized neurological examination (1). The standards developed for to constitute a common language among the professionals constitute a common language for professionals dealing with this disease, and they also provide standardization in scientific studies and comparisons of data among health centers.

The International Standards for Neurological Classification of Spinal Cord Injury (ISNCSCI), which was revised by the American Spinal Injury Association (ASIA) in 2011 and was also accepted by the International Spinal Cord Society, is undoubtedly the most commonly used and most developed classification and has the highest sensitivity among existing scales (2).

In this review, the definitions and terms included in the ISNCSCI booklet and their current usages are explained.

\section{Definitions}

Tetraplegia (preferred over quadriplegia)

This term refers to the motor and/or sensory function loss or impairment in the cervical segments of the spinal cord, secondary to the injuries of neural structures in the spinal canal.
Tetraplegia results in dysfunction in the arms, trunk, legs, and pelvic organs. It does not include brachial plexus lesions or peripheral nerve injuries outside the neural canal.

\section{Paraplegia}

This term refers to motor and/or sensory function loss or impairment in the thoracic, lumbar, or sacral (not cervical) segments, secondary to the injuries of neural structures in the spinal canal.

In paraplegia, arm functions are protected. Depending on the injury level, the trunk, legs, and pelvic organs may be affected. Moreover, this term is used for cauda equine and conus medullaris injuries. It does not include lumbosacral plexus lesions or peripheral nerve injuries outside the neural canal.

\section{Tetraparesis and Paraparesis}

The use of these terms is not recommended because they unclearly refer to incomplete injuries, and they incorrectly suggest that the terms of tetraplegia and paraplegia can be used only for neurologically complete injuries. Instead, the ASIA impairment scale provides an approach that more clearly defines the severity of injury (e.g., completeness). 


\section{Dermatome}

This term defines the area of the skin innervated by the sensory axons of each segmental nerve (root).

\section{Myotome}

This term refers to the collection of muscle fibers that are innervated by the motor axons of each segmental nerve (root).

\section{Sensory Level}

It is determined by evaluating 28 key sensation points in each half of the body (right and left). It is the most caudal level at which pin-pricking (differentiation of dull and sharp) and lighttouch sensations are normal. It can be different in the right and left sides of the body. The most rostral level is used when referring to a single level.

\section{Motor Level}

It is determined by evaluating the key muscle function included in 10 myotomes in each half of the body.

It is defined as the lowest myotome with at least a muscle strength of 3 (by manual muscle testing) provided that the muscles above the level are healthy (with the muscle strength of 5 by manual muscle testing). It may differ in the right and left sides of the body. The most rostral level is used when referring to a single level.

\section{Neurological Injury Level (NIL)}

Provided that sensory and motor functions are normal (intact), NIL refers to the most caudal level at which the muscle strength against sensation and gravity is normal in both the sides of the body.

The segments in which normal function is preserved after sensory and motor evaluation can differ in the right and left sides of the body. Four different segments, including the right sensory, left sensory, right motor, and left motor, are identified for determining neurological level. In contrast, the single NIL of a patient is the most rostral level.

\section{Skeletal Level}

It is the level at which the greatest vertebral damage is observed on radiological examination. The term skeletal level is not included in the recently published International Standards for Neurological Classification of Spinal Cord Injury (ISNCSCI) because bone damage does not always coexist with the spinal cord injury or bone damage level and neurological damage level of the spinal cord are not always consistent with each other.

\section{Sensory Score}

This term refers to the total score that is obtained as a result of a numerical scoring of sensory function. Maximum total score for each of the superficial sensation and pinprick sensation (dull/sharp) is 56 , and total sensory score for each half of the body is 112 .

It reveals the degree of neurological damage accompanying spinal cord injury.

\section{Motor Score}

This term refers to the total score that is obtained as a result of a numerical scoring of motor function.

Maximum score for both extremities is 25 . The total score of the lower and upper extremities is 50 for each one. This score can express the degree of neurological damage that coexists with spinal cord injury.

\section{Incomplete Injury}

This term is used in the presence of preserved sensory and/or motor functions, including the lowest sacral segments (S4-S5) below the neurological level (in the presence of sacral preservation).

Sensory sacral preservation means the existence of sensory protection (intact or impaired) with light touching or pin pricking in one or two sides of the anal musculocutaneous junction (S4S5 dermatome) or the existence of deep anal pressure.

Motor sacral preservation refers to voluntary contraction of the external anal sphincter in digital rectal examination.

\section{Complete Injury}

This term is used when there is no sensory and motor function protected in the lower sacral segments (S4-S5) (for example, in the absence of sacral protection) (3).

\section{Partially Preserved Area (PPA)}

This term is used only for complete injuries, and it defines partially innervated dermatomes and myotomes below sensory and motor levels. PPA must be separately recorded as four different levels for sensory and motor functions and right and left sides (right sensory, left sensory, right motor, and left motor). In the presence of protection in more than one segment, the most caudal segment is recorded.

Peer-review: This manuscript was prepared by the invitation of the Editorial Board and its scientific evaluation was carried out by the Editorial Board.

Conflict of Interest: No conflict of interest was declared by the author.

Financial Disclosure: The author declared that this study has received no financial support.

\section{References}

1. Kirshblum S, Donovan WH. Neurologic assessment and classification of traumatic spinal cord injury. In: Kirshblum S, Campagnolo DI, DeLisa JA, editors: Spinal Cord Medicine. Philadelphia, Lippincott Williams Wilkins, 2002, p.82-95.

2. Kirshblum SC, Burns SP, Biering-Sorensen F, Donovan W, Graves DE, Jha $A$, et al. International standards for neurological classification of spinal cord injury (revised 2011). J Spinal Cord Med 2011;34:535-46. [CrossRef]

3. Waters RL, Adkins RH, Yakura JS. Definition of complete spinal cord injury. Praplegia 1991;9:573-81. [CrossRef] 